\title{
Kinetochore dynein generates a poleward pulling force to facilitate congression and full chromosome alignment
}

\author{
Yan $\mathrm{Li}^{1, *}$, Wei Yu ${ }^{1, *}$, Yun Liang ${ }^{1}$, Xueliang $\mathrm{Zhu}^{1}$ \\ ${ }^{1}$ Laboratory of Molecular Cell Biology, Institute of Biochemistry and Cell Biology, Shanghai Institutes for Biological Sciences, Chi- \\ nese Academy of Sciences, Shanghai 200031, China
}

\begin{abstract}
For proper chromosome segregation, all kinetochores must achieve bipolar microtubule (MT) attachment and subsequently align at the spindle equator before anaphase onset. The MT minus end-directed motor dynein/dynactin binds kinetochores in prometaphase and has long been implicated in chromosome congression. Unfortunately, inactivation of dynein usually disturbs spindle organization, thus hampering evaluation of its kinetochore roles. Here we specifically eliminated kinetochore dynein/dynactin by RNAi-mediated depletion of ZW10, a protein essential for kinetochore localization of the motor. Time-lapse microscopy indicated markedly-reduced congression efficiency, though congressing chromosomes displayed similar velocities as in control cells. Moreover, cells frequently failed to achieve full chromosome alignment, despite their normal spindles. Confocal microcopy revealed that the misaligned kinetochores were monooriented or unattached and mostly lying outside the spindle, suggesting a difficulty to capture MTs from the opposite pole. Kinetochores on monoastral spindles were dispersed farther away from the pole and exhibited only mild oscillation. Furthermore, inactivating dynein by other means generated similar phenotypes. Therefore, kinetochore dynein produces on monooriented kinetochores a poleward pulling force, which may contribute to efficient bipolar attachment by facilitating their proper microtubule captures to promote congression as well as full chromosome alignment.
\end{abstract}

Keywords: kinetochore; cytoplasmic dynein; chromosome; mitosis; force

Cell Research (2007) 17:701-712. doi: 10.1038/cr.2007.65; published online 7 August 2007

\section{Introduction}

Chromosome motions to the spindle midzone, or congression, is an efficient process in mitosis. After nuclear envelope breakdown (NEBD) at prometaphase, the kinetochore, an organelle at the centromere region of each chromatid, is exposed to and thus capable of capturing the spindle MTs. A kinetochore that has successfully captured an MT laterally is usually subjected to rapid poleward

*These two authors contributed equally to this work

Correspondence: Xueliang Zhu

Tel: +86-21-54921406; Fax: +86-21-54921011

E-mail: xlzhu@sibs.ac.cn

Received 6 July 2007; Revised 16 July 2007; Accepted 17 July 2007; published online 7 August 2007
(P) motion. It is believed that the P movement enables the kinetochore to run into more MTs emanated from the approaching pole to facilitate the stable, or "end-on", MT attachment $[1,2]$. Chromosomes with such monooriented kinetochores then experience oscillations toward $(\mathrm{P})$ and away (AP) from their attaching pole until their unattached kinetochores capture MTs from the opposite pole. The now bi-oriented chromosomes move towards the metaphase plate and finally align there, waiting for inactivation of the spindle checkpoint to allow anaphase onset [1-3]. For monooriented kinetochores, their P movement is attributed to the poleward pulling force at the attached kinetochore, whereas their AP movement is likely a composite effect of both the pushing force at the kinetochore and the polar ejection force acting on chromosome arms through nonkinetochore MTs $[2,3]$. These antagonizing forces appear to act alternatively to enable the oscillation, during which kinetochore MTs (k-fibers) remain attached but assemble 
and disassemble accordingly [2, 3].

Full chromosome alignment is a prerequisite for equal segregation of sister chromatids into daughter cells. The spatial location of each chromosome must differ from cell to cell after NEBD. Therefore, it is amazing that usually metaphase is accomplished within $20 \mathrm{~min}$ [4]. How cells achieve chromosome alignment so efficiently is still not fully understood.

Cytoplasmic dynein, a minus-end-directed MT-based motor, is a large complex containing two heavy chains (DHC), two intermediate chains (DIC), four light-intermediate chains, and several light chains $[5,6]$. Another multiprotein complex, dynactin, increases the processivity of dynein and anchors dynein to certain cargos or target sites. Both complexes are believed to link together through the interaction between DIC and p150 $0^{\text {glued }}$, the largest subunit of dynactin [5-7].

Dynein is widely involved in cellular functions requiring MT-based motility, for instances, mitosis, cell migration, and cargo transport $[5,8,9]$. A pool of dynein is located to the kinetochore in $M$ phase through dynactin $[10,11]$, whereas dynactin is recruited to kinetochores by the ZW10/ Rod complex $[12,13]$. There dynein/dynactin can transport kinetochore proteins such as Mad2, BubR1, CENP-E, mitosin (CENP-F), and Rod to spindle poles along k-fibers [14-17]. Although the physiological role of such transport is not fully understood, at least part of it is to inactivate the spindle checkpoint, a mechanism assuring proper timing for anaphase onset $[2,14]$.

Kinetochore dynein is generally believed to play a role in congression by driving the poleward kinetochore movement $[1,2,18]$. Experimental results, however, are controversial. Dynein inactivation before prometaphase by excess dynactin subunit p50 or anti-dynein antibodies indeed blocks the prometaphase/metaphase transition. However, since dynein is also important for spindle organization [19-21], spindle organization is also affected [11, 22]. It is thus difficult to discriminate the kinetochore-specific roles of dynein. On the other hand, microinjecting anti-dynein antibodies or purified p50 in prometaphase cells does not seem to affect congression, though tension is significantly reduced $[14,22]$.

Dynein is recruited to kinetochores by dynactin that binds the ZW10/Rod complex [12]. ZW10 may thus serve as an ideal target for regional elimination of dynein/dynactin. Studies in $z w 10$-null Drosophila mutants indeed indicate reduced poleward chromosome movement in meiosis [23]. In this study, we tried this approach to assess the role of kinetochore dynein in congression. We demonstrate that kinetochore dynein is required for efficient congression and full chromosome alignment. Moreover, dynein generates at monooriented kinetochore a poleward pulling force that is important for chromosome oscillations. We propose that the pulling force contributes to efficient bipolar attachment by facilitating proper microtubule capture of monooriented kinetochores.

\section{Materials and Methods}

\section{Plasmid constructs}

Plasmids for GFP-tagged H2B, p50 $0^{\text {dynamitin }}$, Nudel, and the dynein binding-defective mutant Nudel $^{\mathrm{C} 36}$ were described previously [17, 24, 25]. To express H2B-RFP, the GFP coding region was replaced with mRFP cDNA which was kindly provided by Dr E Fuchs (Rockefeller University, USA).

Plasmids for RNAi were constructed using the vector pTER+ [26]. pTER-ZWi and pTER-Nudi were used to knock down ZW10 and Nudel, respectively [17, 27], whereas either the vector or pTERLuci, which was designed to knock down firefly luciferase [28], served as a control [17]. For time-lapse studies using HeLa cells, the shRNA-expressing module of pTER or pTER-ZWi was cloned into $\mathrm{pH} 2 \mathrm{~B}-\mathrm{RFP}$, independent of the H2B-RFP-expressing module, to form pTER-H2B-RFP or pTER-ZWi-H2B-RFP. In this way, transfectants positive for RFP should also express shRNA.

\section{Cell culture and transfection}

HEK293T and HeLa cells were cultured as described [27]. For protein overexpression, cells were transfected for $48 \mathrm{~h}$ using calcium phosphate method. HeLa cells stably expressing GFP- $\alpha$-tubulin were cloned after G418 selection. Transfections for RNAi were carried out in 35-mm dishes using Lipofectamine 2000 (Invitrogen) for HEK293T cells or Fugene 6 (Roche) for HeLa cells. Cells were transfected for $72 \mathrm{~h}$ to knock down ZW10 [17]. To transiently label chromosomes with H2B-GFP for time-lapse studies, pEGFP-H2B was cotransfected with the RNAi construct or control construct at a ratio of 1:200 into HEK293T cells. H2B-GFP thus also served as a transfection marker. For transfecting HeLa cells stably expressing GFP- $\alpha$-tubulin, pTER-RFP-H2B and pTER-ZWi-RFP-H2B were used instead of pEGFP-H2B and transfection was performed at a ratio of 1:50. To knock down Nudel, two rounds of transfection was performed as described [17].

\section{Antibodies}

Anti- $\alpha$-tubulin mAb was purchased from Sigma-Aldrich. Antip150 glued and BubR1 mAbs were from BD Transduction Laboratories. Chicken anti-Nudel IgY was prepared by using bacterially-expressed Nudel as antigen [17]. Polyclonal antibodies to the CREST antigen were gifts from K.H. Choo (Royal Children's Hospital, Australia). Antibodies against human ZW10 and Rod were kindly provided by Dr. G. Chan (University of Alberta, Canada). Secondary antibodies labeled with Alexa 488, 546, and 647 were purchased from Invitrogen.

\section{Fluorescence staining and microscopy}

Kinetochore staining was performed as described [17]. To disassemble MTs, cells were treated with nocodazole $(10 \mu \mathrm{g} / \mathrm{ml})$ for $1 \mathrm{~h}$ before fixation. Proper antibody combinations were used for multicolor staining, whereas GFP or RFP fusion proteins were visualized directly through the autofluorescence. Nuclear DNA was stained with 4,6-diamidino-2-phenylindole (DAPI). To induce monoasters, cells were treated with monastrol $(50 \mu \mathrm{M})$ (Sigma-Aldrich) for $4 \mathrm{~h}$ [29].

Laser confocal microscopy was performed with the Leica SP2 
system as described for fixed cells [17]. Time-lapse microscopy was performed at $37^{\circ} \mathrm{C}$ using a Leica AS MDW system with a HCX PL APO $63 \times 1.30$ GLYC CORR $37^{\circ} \mathrm{C}$ objective and a CoolSNAP HQ CCD camera (Roper Scientific) [17]. Cells were grown on glass coverslips and cultured in Leibovitz's (L-15) based media supplemented with 10\% fetal bovine serum (Invitrogen) and $7 \mathrm{mM}$ HEPES (pH 7.2).

Quantitation for kinetochore fluorescence intensities was done as described [17]. To reduce influences of quenching and different spatial distribution, only kinetochores showing brighter staining than most of the remaining ones were quantitated in each cell. The average intensity from control cells was set at 10 , while that of experimental cells was made proportional to this value to obtain the relative intensity. Statistic data were presented as mean \pm s.e.m.

The kinetochore-pole distance was measured using NIH image J software. To quantify chromosome motions on monoastral spindles, time-lapse image sequences were imported into image J. The inner edge of a peripheral chromosome was tracked frame-by-frame using the image $\mathrm{J}$ manual-tracking tool. The centroid of the cell was picked using the measure tool. Its distance to the inner edge was then measured in each frame using the software. Data were then analyzed using Microsoft Excel.

\section{Results}

Silencing mammalian ZW10 leads to chromosome misalignment and tension reduction

To assess possible functions of kinetochore dynein in congression, we first tried to influence only this pool of dynein by knocking down ZW10 expression via RNAi. We have previously used an RNAi construct, pTER-ZWi, to silence ZW10 expression in HEK293T cells [17]. Compared to the strong kinetochore staining of ZW10 in cells transfected for $72 \mathrm{~h}$ with a control construct, pTER-Luci [17], the protein was virtually undetectable after transfection of pTER-ZWi (Figure 1A-B). Consistent with previous reports $[13,30,31]$, dynactin (indicated by $150^{\text {glued }}$ ) and
A
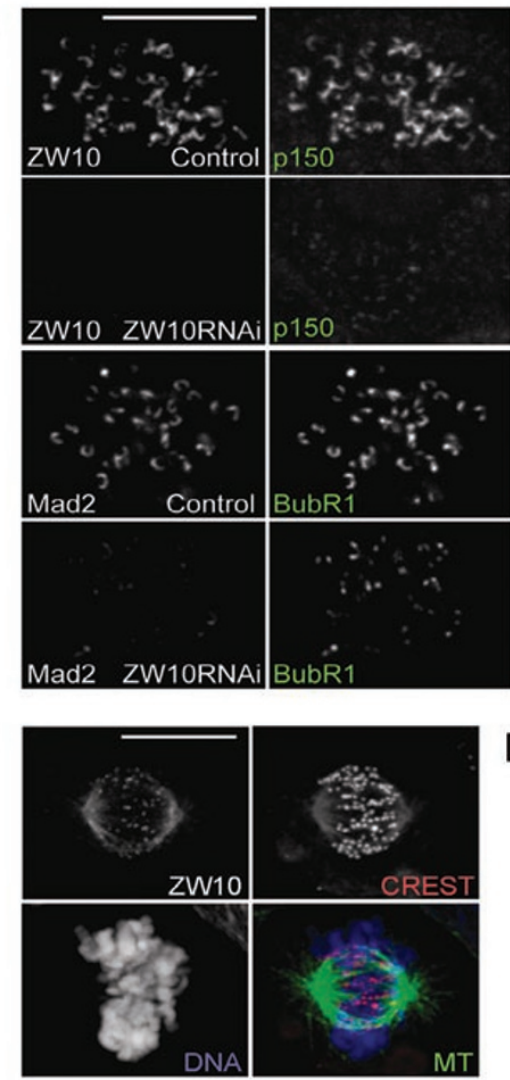
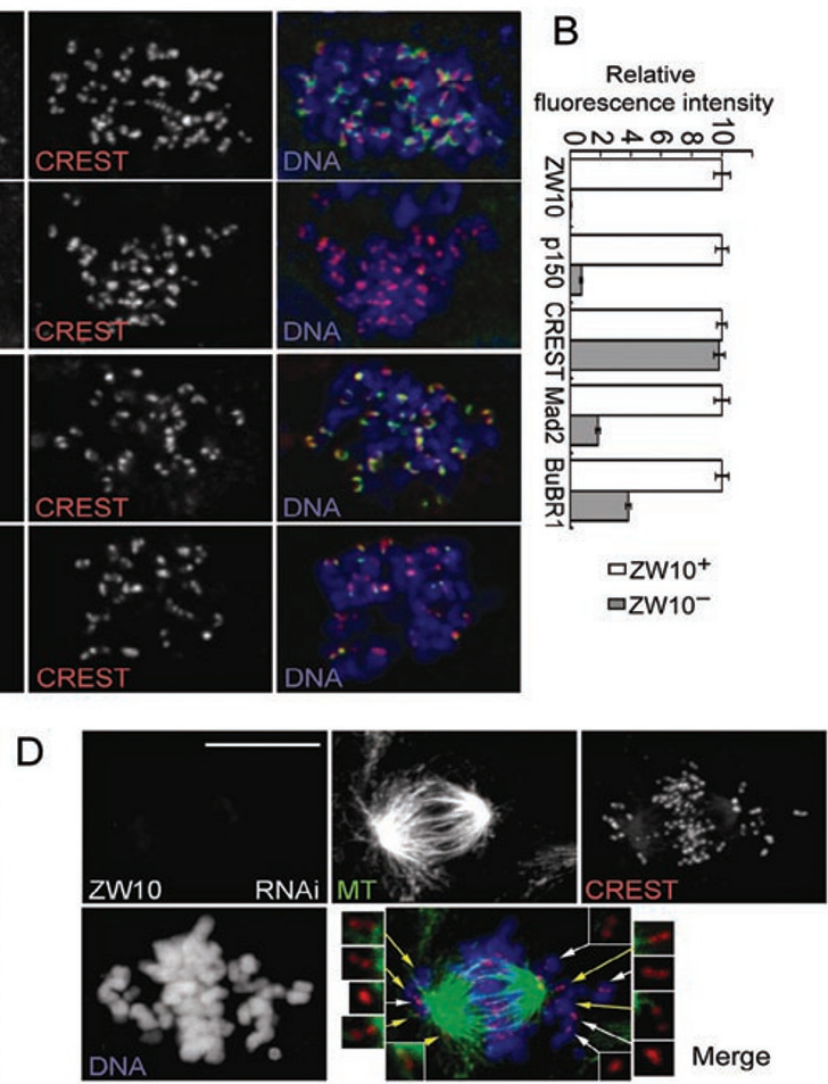

Merge

Figure 1 Silencing ZW10 expression induces chromosome misalignment. (A-B) ZW10 depletion reduces kinetochore dynactin, Mad2, and BubR1. HEK293T cells transfected with pTER-Luci (control) or pTER-ZWi (RNAi) were fixed $72 \mathrm{~h}$ post transfection after nocodazole treatment for 1 hour. The indicated proteins were immunostained with appropriate antibodies. Chromosomal DNA was stained with DAPI. Quantitation results $(n=150)$ are presented as mean \pm s.e.m. (C) Typical configuration of control cells in late prometaphase. (D) A typical ZW10-depletion cell in pseudometaphase. Insets are $2 \times$ magnifications from proper optical layers to show MT-kinetochore relationship of misaligned chromosomes. Scale bars, $10 \mu \mathrm{m}$. 
Mad2 were decreased by $92.7 \%$ and $81.7 \%$, respectively, from the kinetochore, whereas BubR1 showed less reduction (61.7\%) and was still visible (Figure 1A-B).

Similar to antibody microinjection to inactivate ZW10 in HeLa cells [32], ZW10 depletion in HEK293T cells by RNAi also frequently led to misaligned chromosomes. Of ZW10-depleted cells in prometaphase or metaphase, $23.9 \%(\mathrm{n}=260)$ were in pseudometaphase, a metaphase-like state with misaligned chromosomes [33]. Most misaligned chromosomes were clustered near the spindle pole on the astral side and appeared monooriented or unattached, as judged through their MT-kinetochore relationships (Figure 1D). In comparison, pseudometaphase cells occupied only $5.8 \%(n=299)$ in matched control populations. Moreover, misaligned chromosomes in control cells were usually distributed between the poles, suggesting that they were in the process of congression. Therefore, distribution of unattached and monooriented chromosomes in the astral region in ZW10-depleted pseudometaphase cells suggests defects in formation of bi-oriented chromosomes.

ZW10 depletion also resulted in decreased tension across sister kinetochores. In control transfectants, the interkinetochore distance was $1.79 \pm 0.01 \mu \mathrm{m}$ on average $(n=110)$ for fully aligned chromosomes. The value for unaligned chromosomes in early prometaphase, i.e., when kinetochores were not subjected to tension, was $1.01 \pm 0.01$ $\mu \mathrm{m}$. In ZW10-depleted cells, the interkinetochore distance was $1.31 \pm 0.02 \mu \mathrm{m}$ for aligned chromosomes, indicating a tension reduction by $61.5 \%$ [34-36].

\section{Silencing $Z W 10$ results in a severe delay in both congres- sion and anaphase onset}

To understand how chromosome misalignment occurred in the absence of ZW10, we monitored chromosome behaviors in mitotic HEK293T cells using H2B-GFP as a chromosome marker [17, 37]. H2B-GFP-positive cells cotransfected with pTER-Luci $(n=16)$ showed normal mitotic progressions when imaged from prophase or early prometaphase (Figure 2A; Table 1; for reference, see Supplementary information, Video 1). All of them started anaphase within $1 \mathrm{~h}$. In contrast, such normal cases were rare $(1 / 26)$ in H2B-GFP-positive cells cotransfected with pTER-ZWi. Instead, $30.8 \%$ of mitotic transfectants $(8 / 26)$ failed to start anaphase in $3 \mathrm{~h}$. Although the remaining $65.4 \%(17 / 26)$ initiated anaphase to form two $(7 / 26)$ or more (10/26) daughter nuclei after considerably prolonged delay, the average time from prometaphase to anaphase onset was 3.1-fold longer than that of control cells (Figure

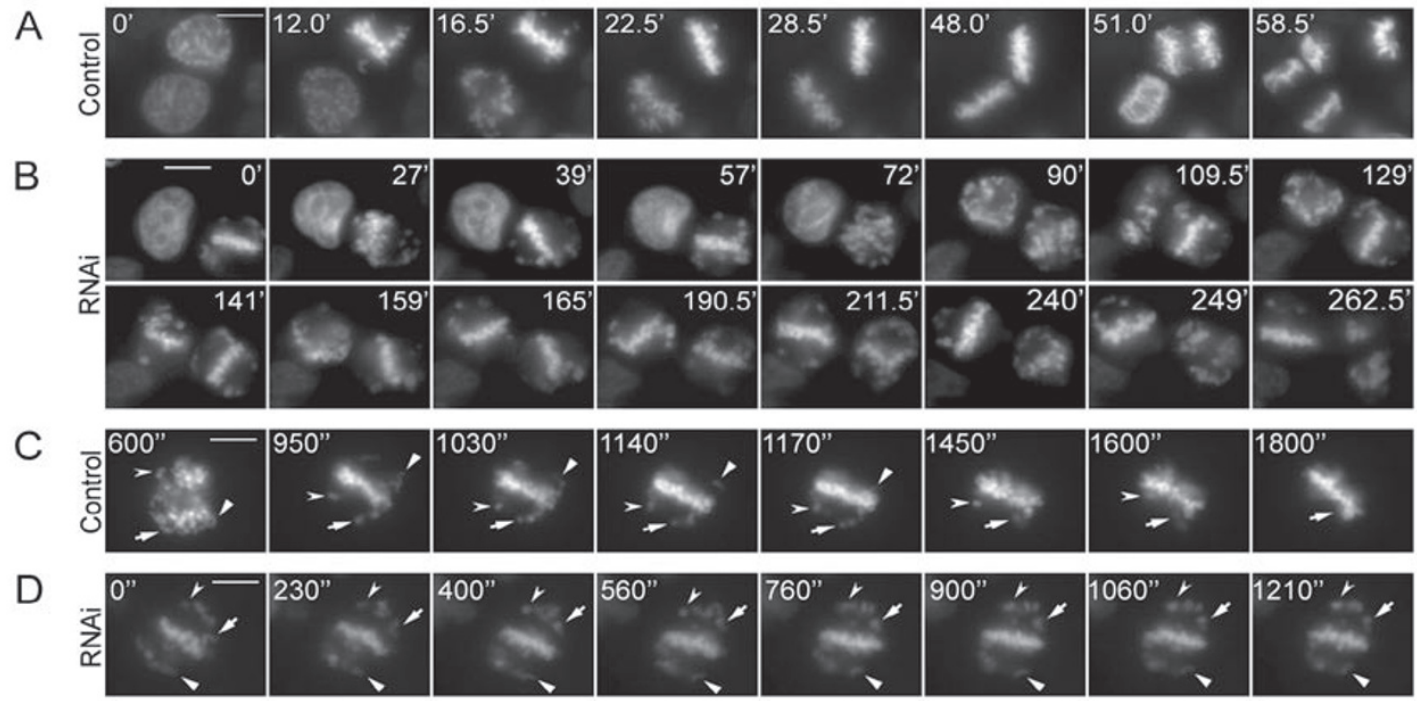

Figure 2 ZW10 depletion results in delayed anaphase onset. A plasmid for expression of H2B-GFP as both transfection and chromosome markers was cotransfected into HEK293T cells at 1:200 ratio with either pTER-Luci (control) or pTER-ZWi (RNAi). GFPpositive mitotic cells were monitored after $72 \mathrm{~h}$. Scale bars, $10 \mu \mathrm{m}$. (A) Representative frames showing normal mitotic progression of control cells. Cells were imaged at 1.5-min intervals. For reference, see Supplementary information, Video 1. (B) Abnormal mitotic progressions of ZW10-depleted cells. Note that the cell on the right started anaphase at 240 min even in the presence of many unaligned chromosomes. Also see Supplementary information, Video 2. (C) Efficient chromosome congression in control cells. Cells were imaged at 10-second intervals to visualize detailed chromosome movement. The indicated chromosomes congress relatively slowly. Also see Supplementary information, Video 1. (D) ZW10 depletion impairs congression of unaligned chromosomes. Three representative chromosomes are indicated. Also see Supplementary information, Video 3. 
Table 1 Summery for time-lapse assays on HEK293T cells

\begin{tabular}{lcccccc}
\hline RNAi & $\begin{array}{c}\text { Total } \\
\mathrm{n}\end{array}$ & $\begin{array}{c}\text { Divisions in } \\
\text { pro- } \\
\text { metaphase }(\mathrm{n})\end{array}$ & $\begin{array}{c}\text { Divisions in } \\
\text { pseudo- } \\
\text { metaphase }(\mathrm{n})\end{array}$ & $\begin{array}{c}\text { Divisions in } \\
\text { metaphase } \\
(\mathrm{n})\end{array}$ & $\begin{array}{c}\text { Visible metaphase } \\
\text { plate }(\min )^{\mathrm{a}}\end{array}$ & $\begin{array}{c}\text { Anaphase onset } \\
(\min )^{\mathrm{a}}\end{array}$ \\
\hline Luc & 16 & 0 & 0 & 16 & $6.0 \pm 0.5(\mathrm{n}=16)$ & $39.0 \pm 2.7(\mathrm{n}=16)$ \\
ZW10 & 26 & 8 & 8 & $2^{\text {b }}$ & $28.2 \pm 3.8(\mathrm{n}=9)$ & $120.6 \pm 15.0(\mathrm{n}=17)$ \\
\hline
\end{tabular}

${ }^{\mathrm{a}}$ Time was counted from prometaphase;

${ }^{\mathrm{b}}$ One divided at $114 \mathrm{~min}$; another appeared normal and was excluded from the subsequent analysis.

2B; Table 1; Supplementary information, Video 2). A larger difference was actually expected because most pTER-ZWi transfectants $(22 / 26)$ were recorded from prometaphase and thus might have already been in this phase for some time before being imaged. Therefore, ZW10 depletion appears to impair but not abolish the spindle checkpoint.

The mitotic delay was attributed to chromosome misalignment. In control cells, the prometaphase/metaphase transition only took $17 \mathrm{~min}$ on average $(\mathrm{n}=16)$ (Figure 2A; data not shown). Nevertheless, $61 \%$ of pTER-ZWi transfectants $(16 / 26)$ stayed in a prometaphase-like state before anaphase onset or termination of the experiment (Table 1). $34 \%$ of transfectants $(9 / 26)$ needed 4.7 -fold longer time to form a visible metaphase plate (Table 1). Moreover, full chromosome alignment was difficult to achieve (Figure 2B; Supplementary information, Video 2).

For more detailed view of chromosome movement, mitotic cells were imaged every 10 seconds. Control cells still divided within $1 \mathrm{~h}$ under such condition (Supplementary information, Video 1). Moreover, after a metaphase plate was visible, unaligned chromosomes were able to fully congress within $20 \mathrm{~min}(\mathrm{n}=15)$ (Figure 2C; Supplementary information, Video 1). In ZW10-depleted cells at pseudometaphase $(\mathrm{n}=14)$, however, full chromosome alignment was not achieved during the same period of time (Figure 2D; Supplementary information, Video 3). Only occasionally did misaligned chromosomes move plateward in ZW10-depleted cells (Figure 2D; data not shown). When congressing chromosomes, which were presumably bi-oriented, were tracked, their mean velocity was $4.32 \pm 0.16 \mu \mathrm{m} / \mathrm{min}(\mathrm{n}=17)$. This value was similar to that in control cells $(4.39 \pm 0.17 \mu \mathrm{m} / \mathrm{min} ; \mathrm{n}=45)$. Therefore, ZW10 depletion did not affect congression of bi-oriented chromosomes. This is consistent with the observation that the misaligned chromosomes were either monooriented or unattached in fixed cells lacking ZW10 (Figure 1D).

Taken together, these results indicate that ZW10 depletion results in severe defects in the prometaphase/metaphase transition probably due to difficulties for monooriented chromosomes to establish bipolar attachment.
ZW10 depletion does not disrupt spindle organization and function

The MT-kinetochore attachment is affected by both spindle and kinetochore. Although ZW10 deficiency in Drosophila did not influence spindle morphology [38], we still assessed this issue. In fixed samples, $80.0 \%$ of ZW10depleted HEK293T cells in prometaphase or metaphase $(\mathrm{n}=242)$ had normal-shaped bipolar spindles (Figure 1D), which was similar to the value $(87.2 \% ; n=299)$ for control cells.

To further corroborate this, we monitored spindle organization in live cells stably expressing GFP- $\alpha$-tubulin. Since HEK293T cells were G418-resistent, we used HeLa cells to screen for stable cell lines. To increase RNAi efficiency and at the same time avoid high-level expression of H2B, pTER-ZWi-H2B-RFP was contransfected with pTER-ZWi at a ratio of 1:50. Similarly, control cells were cotransfected using pTER-H2B-RFP and pTER. Timelapse microscopy indicated that HeLa cells transfected with pTER-ZWi-H2B-RFP (Figure 3; Table 2) behaved similarly to ZW10-depleted HEK293T cells (Figure 2). The average time from NEBD to anaphase onset among 11 control cells was $41.5 \pm 4.2 \mathrm{~min}$ (Table 2). Of 18 pTER-ZWi transfectants monitored, however, only 3 cells exhibited normal M phase progression. The remaining 15 cells showed considerably delayed division (Table 2). Nevertheless, these 15 cells showed virtually normal spindle organization during the course of M phase progression (Figure 3; Supplementary information, Video 4), except for a tiny extra pole of one cell after $105 \mathrm{~min}$. Despite normal spindles, congression was markedly delayed, as chromosomes needed a 2.2 -fold longer time to form a clear metaphase plate (Figure 3; Table 2). Moreover, all these cells displayed difficulty in full chromosome alignment (Figure 3; data not shown). Thus, the phenotypes in ZW10-depleted cells are attributed to defects at the kinetochore.

Dynein inactivation is correlated with congression defects and chromosome misalignment

If kinetochore dynein had a role in congression, its inac- 
A
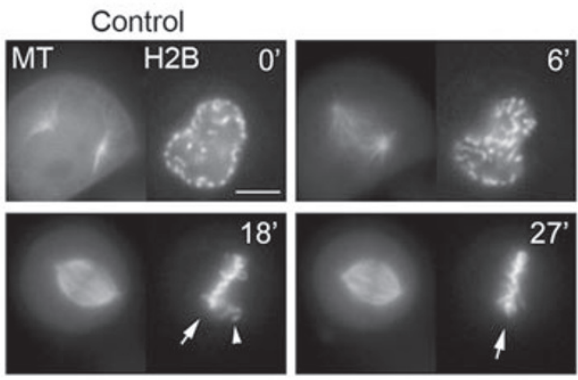

B
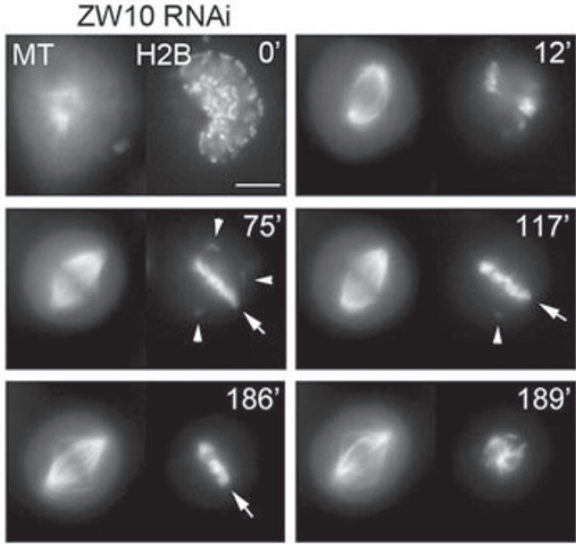
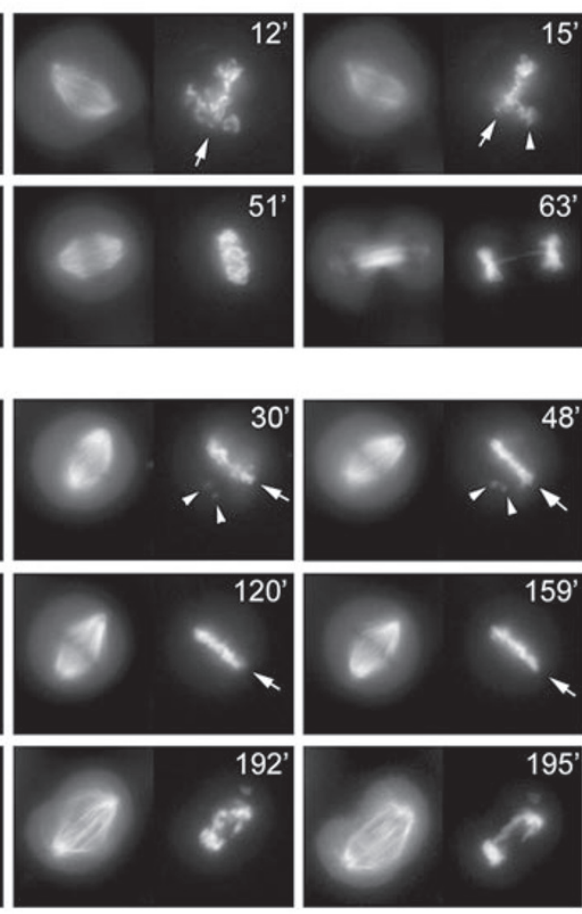

Figure 3 ZW10 is not required for spindle organization and function. HeLa cells stably expressing GFP- $\alpha$-tubulin (MT) were transiently transfected with either pTER (control) or pTER-ZWi (RNAi) for $72 \mathrm{~h}$, with H2B-RFP (H2B) as both transfection and chromosome markers. Early mitotic cells were then imaged at 3-min intervals for up to $4 \mathrm{~h}$. Representative images are shown. Scale bars, $10 \mu \mathrm{m}$. (A) M phase progression of a typical control cell. (B) M phase progression of a typical ZW10-depleted cell. Anaphase was initiated at around $189 \mathrm{~min}$. Arrows indicate the metaphase plate. Arrowheads indicate misaligned chromosomes. Also see Supplementary information, Video 4.

Table 2 Summery for time-lapse assays on HeLa cells

\begin{tabular}{|c|c|c|c|c|}
\hline RNAi & $\begin{array}{c}\text { Total } \\
\mathrm{n}\end{array}$ & $\begin{array}{l}\text { Visible metaphase } \\
\text { plate (min) }{ }^{\text {a }}\end{array}$ & $\begin{array}{l}\text { Prometa-metaphase } \\
\text { transition }(\mathrm{min})^{\mathrm{a}}\end{array}$ & $\begin{array}{c}\text { Anaphase } \\
\text { onset (min) }\end{array}$ \\
\hline Mock & 11 & $10.5 \pm 0.5$ & $18.8 \pm 2.1$ & $41.5 \pm 4.2$ \\
\hline
\end{tabular}

${ }^{\text {a Time was counted from NEBD; }}$

${ }^{\mathrm{b}}$ Only cells without misaligned chromosomes were counted.

tivation by other means would also result in similar defects as ZW10 depletion. Nudel binds DHC [39] and is essential for dynein function $[15,17,24]$. We have previously shown that depletion of Nudel by RNAi or overexpression of a dynein-binding-defective mutant Nudel $^{\mathrm{C} 36}$ inactivates dynein in entire cells with little effect on kinetochore ZW10, Mad2, and BubR1 [17]. Overexpression of the dynactin subunit p50 inhibits dynein function as well by disrupting the integrity of dynactin $[11,17]$. Although these ways of dynein inactivation indeed impaired congression, an apparent disadvantage is that they also result in spindle abnormality $[11,17]$. Therefore, to diminish the influence of spindle configuration on chromosome behaviors, we only checked mitotic cells with bipolar spindles.

We first examined HEK293T cells overexpressing GFP-Nudel ${ }^{\mathrm{C} 36}$ and found that $31.7 \%(n=104)$ of them with bipolar spindles were in pseudometaphase (Figure 4A). In contrast, the value was only $9.3 \%(n=102)$ for cells overexpressing GFP-Nudel. Similar to those in ZW10-depleted cells (Figure 1), misaligned chromosomes in GPF-Nudel ${ }^{\mathrm{C} 36}$ - 


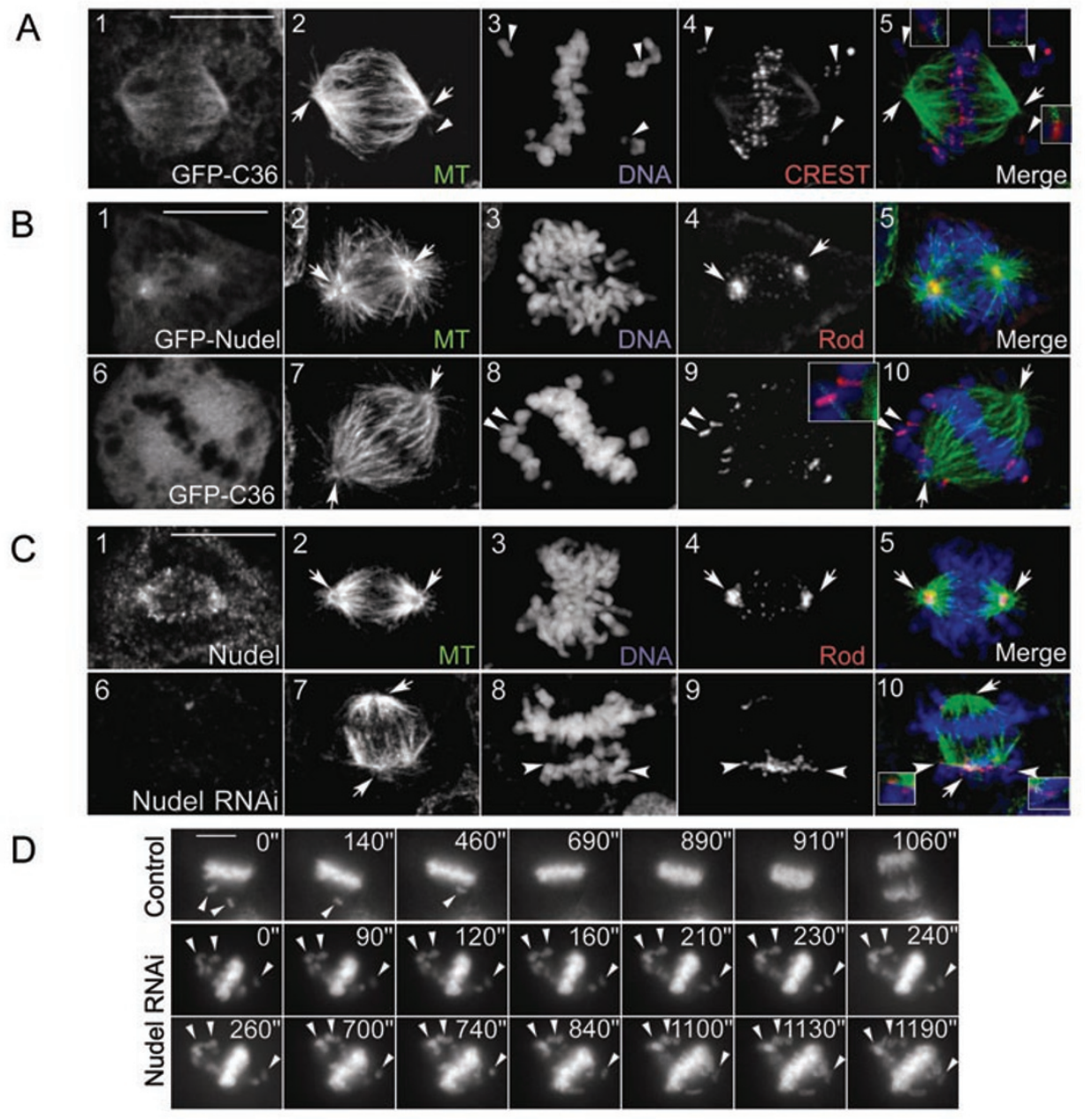

Figure 4 Inactivation of dynein by other means also impairs full chromosome alignment. (A-B) Typical pseudometaphase cells overexpressing GFP-Nudel ${ }^{\mathrm{C} 36}$. HEK293T cells were transfected for $48 \mathrm{~h}$ to express either GFP-Nudel or GFP-Nudel ${ }^{\mathrm{C} 36}$ and processed $^{\mathrm{B}}$ for immunostaining. Insets are $2 \times$ magnifications from proper optical layers to show MT-kinetochore relationship of misaligned chromosomes (arrowheads). Arrows indicate spindle poles. (C) A control cell in late prometaphase and a typical pseudometaphase cell lacking Nudel. Cells were transfected with pTER (panels 1-5) or pTER-Nudi (panels 6-10) and processed for immunostaining. (D) Nudel depletion impairs congression of misaligned chromosomes. A plasmid for expressing H2B-GFP was cotransfected with pTER (control) or pTER-Nudi (RNAi) into HEK293T cells. Late prometaphase cells were imaged at 10-second intervals for 20 min. Representative misaligned chromosomes are indicated by arrowheads. Also see Supplementary information, Video 5. Scale bars, $10 \mu \mathrm{m}$.

overexpressing cells showed monotelic MT attachment or no attachment (Figure 4A). Both the stable ("end-on") and transient ("lateral") attachments were seen (Figure 4A-B, insets). We also noticed that the prominent Rod staining on misaligned chromosomes could serve as a marker for unattached or monooriented kinetochores (Figure 4B, panels $6-10$ ). Similarly, $47.5 \%$ of Nudel-depleted mitotic cells with bipolar spindles $(\mathrm{n}=100)$ were in pseudometaphase (Figure 4C), whereas the value was 9.0\% ( $\mathrm{n}=106)$ for mock-transfected cells. Bright Rod staining was also observed on misaligned chromosomes in Nudel-depleted cells (Figure 4C, panels 6-10), suggesting monotelic MT attachment or no attachment as well.

To understand the behaviors of misaligned/unaligned chromosomes, we monitored living cells in 10 -second intervals for $\sim 20 \mathrm{~min}$, a time normally sufficient for the prometaphase/metaphase transition (Figure 2A). In control cells transfected with pTER $(n=13)$, unaligned chromosomes always moved into the metaphase plate (Figure 4D; for reference, see Supplementary information, Video 1). However, misaligned chromosomes in most pTER-Nudi transfectants $(15 / 17)$ failed to achieve full metaphase 
alignment during recording (Figure 4D; Supplementary information, Video 5). Misaligned chromosomes in most RPF-Nudel ${ }^{\mathrm{C} 36}$ (7/11) or RFP-p50 expressing cells (6/6) failed to achieve full alignment in $20 \mathrm{~min}$ as well (data not shown).

Therefore, chromosome misalignment due to defects in the formation of bipolar attachment is a common phenotype of inactivation of kinetochore dynein.

Kinetochore dynein generates a poleward pulling force that may facilitate bipolar attachment

Dynein has long been suspected to generate the pulling force critical for the P movement of the kinetochore [1]. Its possible contributions to congression have also been speculated $[1,2]$. Although the reduction of tension by $61.5 \%$ upon ZW10 depletion supported the force generation role of dynein, theoretically such a reduction could be a result of increased pushing force at the kinetochore as well.

To verify whether kinetochore dynein generated a poleward pulling force, we made advantages of monoastral spindles, in which kinetochores would be only monotelic and thus their movement would reflect the direction of the net force. Monoastral spindles were induced upon treatment with monastrol, a small-molecule inhibitor of the kinesin motor Eg5 [29, 40]. In monastrol-treated control HEK293T cells, kinetochores were obviously subjected to net poleward pulling force: most kinetochores were oriented proximal to the pole with their chromosome arms significantly stretched backward (Figure 5A, panels 1-5) $[29,41]$. In contrast, chromosomes were short, straight, and scattered in ZW10-depleted cells (Figure 5A, panels 6-10). Their kinetochores were dispersed as well (Figure 5A-B). $53.0 \%$ of them were located farther than $4.5 \mu \mathrm{m}$ away from the poles, compared to $12.6 \%$ in control cells. As k-fibers were obvious in ZW10-depleted cells (Figure 5A, panel 10 , arrowheads), such dispersions were not due to loss of the MT-kinetochore attachment. Rather, they strongly suggested reduced poleward pulling force. Similar results were obtained using Nudel-depleted cells (Supplementary Figure S1A-B).
A
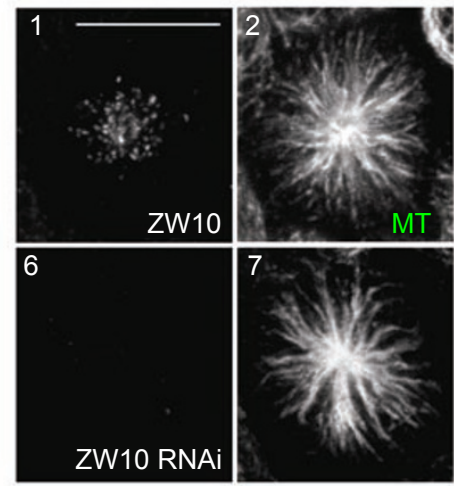

B

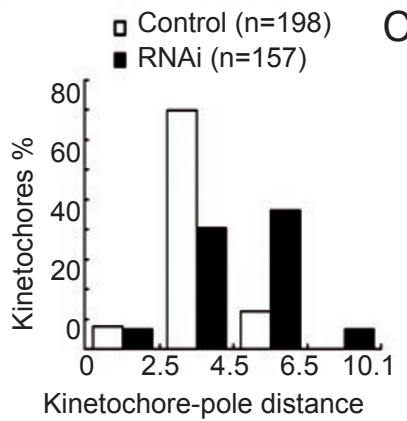

$(\mu \mathrm{m})$
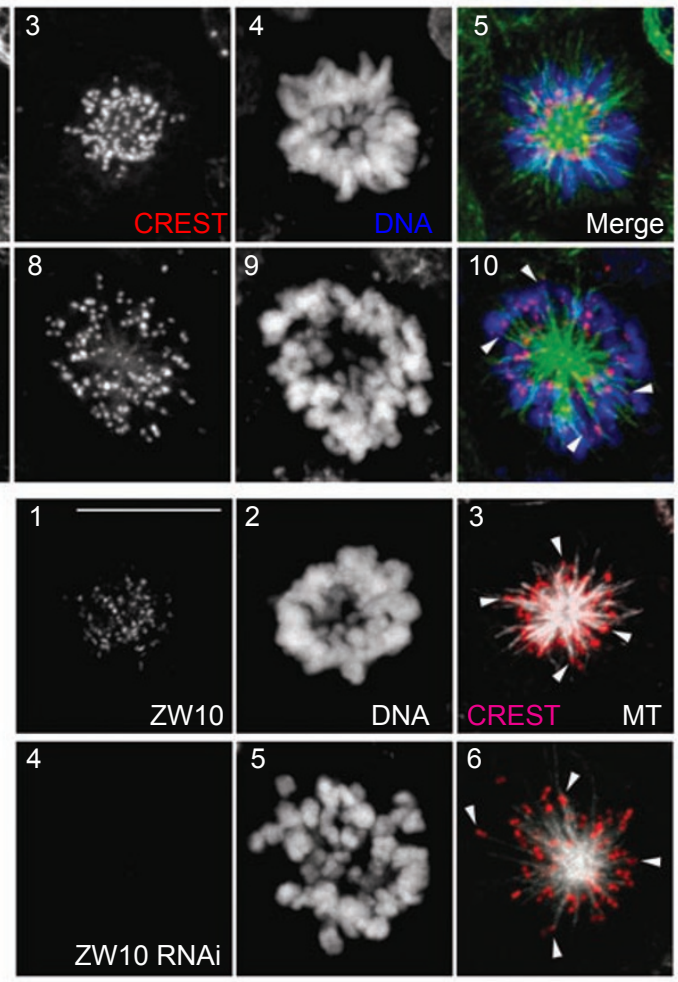

Figure 5 ZW10 depletion results in chromosome dispersion on monoastral spindles. (A) Typical chromosome positioning on monoasters. HEK293T cells transfected for 68 hours with pTER-Luci (panels 1-5) or pTER-ZWi (panels 6-10) were treated with monastrol for 4 hours to induce monoasters, followed by processing for immunostaining. Arrowheads indicate representative kinetochores and their k-fibers. (B) Percentage of kinetochores with kinetochore-pole distances in the indicated ranges. (C) Cells treated as in (A) were incubated with $0.1 \mathrm{mM} \mathrm{CaCl}_{2}$ for 90 seconds to destabilize non-kinetochore $\mathrm{MTs}$ prior to fixation. Arrowheads indicate representative monooriented kinetochores. Scale bars, $10 \mu \mathrm{m}$. 
To exclude the possibility that the chromosome dispersions were due to lack of k-fibers, $\mathrm{Ca}^{2+}$ treatment was performed to destabilize non-kinetochore MTs [29, 41]. In control cells, such a treatment had little effect on the compact distribution patterns of kinetochores, except that the chromosome arms were no longer pushed away from the pole, confirming loss of the polar ejection force (Figure 5C, panels 1-3). Similarly, chromosomes and their kinetochores in ZW10-depleted cells were still dispersed (Figure 5C, panels 4-6). Nevertheless, k-fibers were clearly visible for most chromosomes away from the pole (Figure 5C, panels 4-6). These results further attribute the chromosome dispersion in ZW10-depleted cells to loss of the poleward pulling force.

Finally, to exclude the possibility that the chromosome dispersions were resulted from increased pushing force at the kinetochore or hyperactive oscillation, we monitored the actual chromosome movements in living cells at 10second intervals for $20 \mathrm{~min}$. In control cells with monoastral spindles $(n=17)$, chromosomes were long and exhibited active oscillations towards and away from their centroid (Figure 6A-B; Supplementary information, Video 6). In ZW10-depleted cells $(\mathrm{n}=17)$, such directional oscillations were largely attenuated (Figure 6C-D; Supplementary information, Video 7). Moreover, similar to fixed cells (Figure 5A, panel 9), chromosomes in ZW10-depleted live cells were short (Figure 6C). Taken together, we conclude that kinetochore dynein indeed generates a poleward pulling force critical for $\mathrm{P}$ movement of monooriented chromosomes.

\section{Discussion}

We demonstrate that ZW10 is important for both efficient congression and full chromosome alignment in mammalian cells. ZW10 depletion by RNAi increased the time required to form a visible metaphase plate by 4.7 -fold, indicating reduced congression efficiency. Furthermore, although most chromosomes could eventually align at the metaphase plate after a considerable delay, unaligned chromosomes were frequently seen and full alignment was difficult to achieve (Figures 1, 2). These chromosomes often stayed outside the spindle and were monooriented or unattached (Figure 1). As congressing chromosomes still displayed similar velocities compared to those in control cells (4.32 vs. $4.39 \mu \mathrm{m} / \mathrm{min}$ ) (Figure 2), the major defect was attributed
A
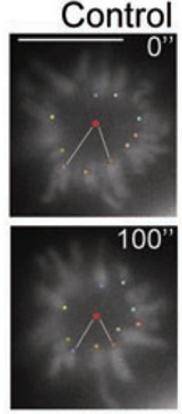

C
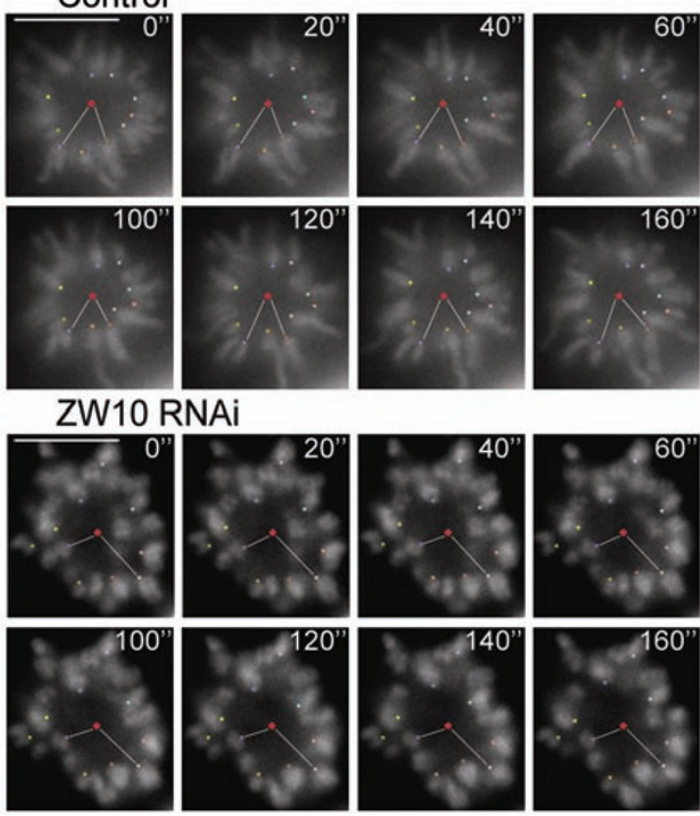
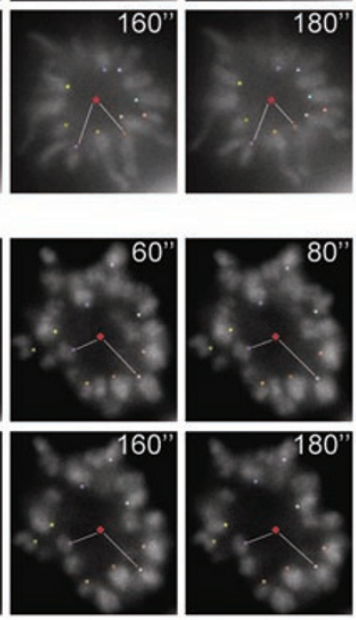

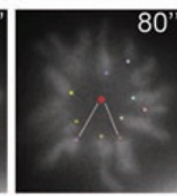

B

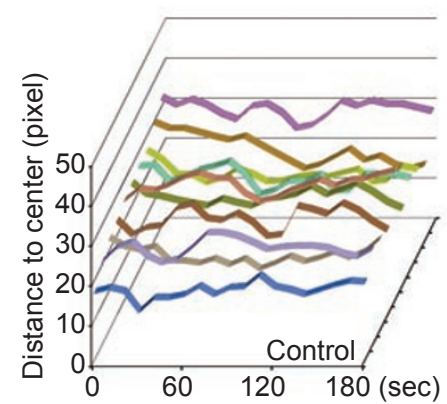

D

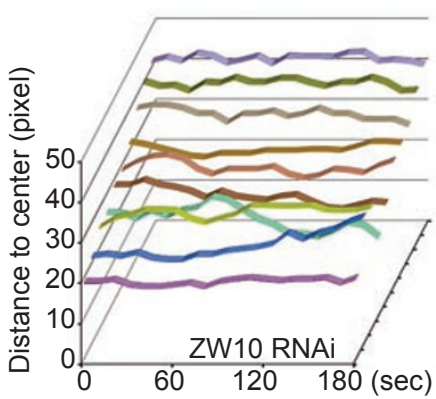

Figure 6 ZW10 depletion attenuates chromosome oscillation on monoasters. Selected frames of a typical control cell (A) and a ZW10-depleted cell (C) during the first 180 seconds of recording are shown. HEK293T cells transfected as in Figure 2 for $68 \mathrm{~h}$ were treated with monastrol for additional $4 \mathrm{~h}$ to induce monopolar spindles. H2B-GFP-positive mitotic cells were then imaged at 10second intervals for $20 \mathrm{~min}$. The innermost side of each of 10 chromosomes is marked with a color dot, and its distance to the cell center (red spot) is measured frame by frame. Data are then presented as a curved line of the same color in (B) or (D), respectively. Also see Supplementary information, Video 6-7. 
to inefficient MT-kinetochore association.

The defect in MT attachment is mainly due to lack of kinetochore dynein. ZW10 depletion significantly represses kinetochore association of the Mad1/Mad2 complex (Figure 1) [30, 31], whereas kinetochore BubR1, mitosin (CENP-F), and Nudel are also partially reduced (Figure 1) $[17,30]$. To rule out possible contributions of these proteins, we inactivated dynein by $\mathrm{Nudel}^{\mathrm{C} 36}$ overexpression or Nudel depletion. Either of the two treatments has been shown to block the prometaphase/metaphase transition, without affecting kinetochore associations of Mad2, BubR1, and mitosin [17]. To diminish the potential effect on spindle, we examined cells with bipolar spindle and found similar phenotypes of chromosome misalignment (Figure 4). Inactivation of dynein by p50 overexpression [11], which only weakly reduces kinetochore localization of Nudel, manifested similar phenotypes as well (data not shown). Nevertheless, as most, sometimes all, chromosomes can eventually align at the spindle midzone in cells lacking dynein activity but with bipolar spindles (Figures 2-4) [11], kinetochore dynein is dispensable for congression of chromosomes with bipolar attachment. This probably explains why microinjection of anti-dynein antibodies into prometaphase cells has little effect on congression [14], since most kinetochores may have established bipolar attachment by the time when the injected antibodies take into effect.

We prove that kinetochore dynein generates a pulling force critical for the poleward movement of monooriented chromosomes. Chromosome oscillations on monoastral spindles were significantly repressed in ZW10-depleted cells (Figure 6). In contrast to the tightly-packed kinetochores and V-shaped chromosomes around the pole of monopolar spindle in control cells, kinetochores in cells lacking either ZW10 or Nudel were scattered, even though they were mostly monooriented, and their chromosomes displayed a short, straight configuration (Figure 5; Supplementary information, Figure S1). Therefore, the dispersions were attributed to lack of the poleward pulling force produced by dynein. Consistently, the reduced tension by $61.5 \%$ in ZW10-depleted cells was also attributed to inactivation of kinetochore dynein. During the preparation of this manuscript, Yang and colleagues reported similar effects of ZW10 RNAi on poleward chromosome movement and congression [42]. Together with results showing that microinjecting an anti-dynein antibody repressed chromosome movement towards the pole of monoaster formed due to depletion of TPX2, they also reached similar conclusion about the force generation role of kinetochore dynein [42]. Nevertheless, their study did not address the issue of chromosome misalignment.

Kinetochore dynein/dynactin may help to increase congression efficiency in multiple ways (Figure 7A) [1, 2]. Chromosomes with syntelic attachment tend to stay away from the spindle [34, 43]. Probably, occupation of their kinetochores by MTs from one pole impedes association with MTs from another pole, whereas pulling forces from two opposing poles are critical for maintaining chromosomes in the spindle. It has been shown that syntelic chromosomes move back to the pole before their congression [43]. This phenomenon on one hand suggests that the poleward movement is critical for correction of the false attachment [43]. On the other hand, moving scattered chromosomes, including stably or transiently-attached monotelic ones (Figure 7A), back to the pole may allow them to reenter the spindle region, as demonstrated in the
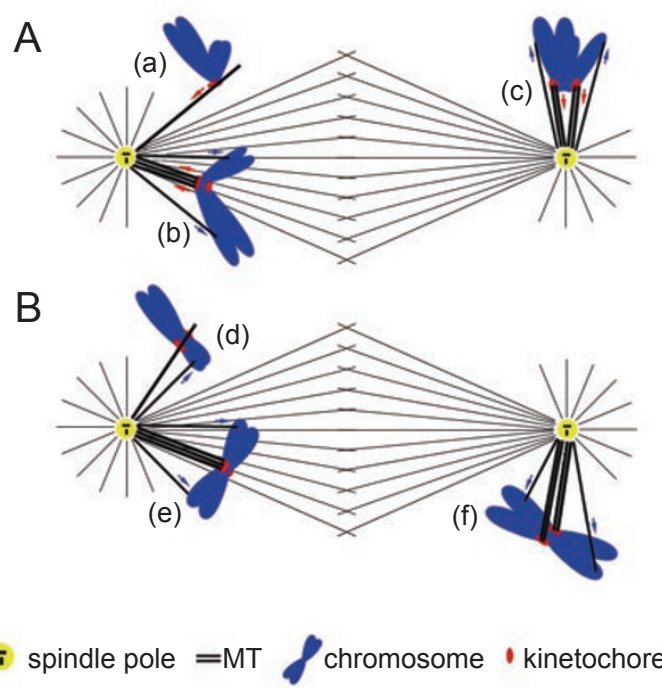

Figure 7 Proposed roles of the poleward pulling force of kinetochore dynein in congression and full chromosome alignment. (A) Monotelic chromosomes in normal cells. (a) The pulling force (red) drives rapid movement of kinetochore along transiently-associated MTs towards the pole to facilitate stable monotelic attachment [1]. (b) The chromosome oscillation powered by the pulling force and the polar ejection force (blue) helps to orient the unattached sister kinetochore towards the opposite pole, increasing its chance of proper MT capture. (c) For chromosome with syntelic attachment, the movement to the attaching pole facilitates its congression [43]. This is probably true as well for other monotelic chromosomes lying outside the spindle. (B) Monotelic chromosomes in cells lacking kinetochore dynein activity. (d) Lack of the poleward movement reduces the efficiency of stable MT attachment. (e) Lack of chromosome oscillation leads to misoriented kinetochores that may hinder proper MT capture of the unattached sister kinetochore. (f) Chromosome with syntelic attachment does not move to the pole. Such chromosomes tend to lie outside the spindle [43], thus are likely to become misaligned. Similarly, monooriented chromosomes may become misaligned as well once they happen to locate outside the spindle. 
case of syntelic chromosomes [43], thus increasing their chance of congression. Yang and colleagues reported that in ZW10-depleted cells K-fiber stability was reduced upon cold treatment [42]. We observed similar results as well in cells lacking Nudel or overexpressing Nudel ${ }^{\mathrm{C} 36}$ (data not shown), thus attributing the effect to the lack of kinetochore dynein activity. Dynein-mediated stabilization of k-fibers may therefore contribute to efficient congression as well [42]. In this context, kinetochores may sometimes lose MT attachment in cells lacking kinetochore dynein activity. Consistent with this, positioning of some unattached misaligned chromosomes near poles in such cells indeed suggests that they are once attached by MTs (Figure 1D; Figure 4).

Moreover, during chromosome oscillation, antagonizing actions of the pulling force produced by kinetochore dynein and the polar ejection force by chromokinesins [44] may help to orient monotelic kinetochores such that the unattached sister kinetochores face the opposite pole to increase the chance of proper MT capture (Figure 7A). In addition to MT capture, the longitudinal orientation of sister kinetochores (Figure 7A) may also minimize formation of false attachments [45] by shielding each sister kinetochore from MTs from opposing poles.

We propose that functions of dynein in increasing congression efficiency may also help to assure full chromosome alignment. For instance, lack of the pulling force will inhibit the poleward movement of chromosomes outside the spindle (Figure 7B), thus leading to chromosome misalignment. Moreover, to make things worse, the polar ejection force of chromokinesins and/or the pushing force of CENP-E may drive these chromosomes further away from the spindle area (Figure 5; Figure 7B). Interestingly, CENP-E is also required for full chromosome alignment $[35,36,46]$, implying requirement of multiple machineries to both guarantee full alignment and prevent chromosome loss effectively. Indeed, neither dynein nor CENP-E is essential for congression per se, since most chromosomes are able to eventually align at either's absence. Dynein is likely only critical for difficult chromosomes, for instance, those with syntelic attachment or somehow laying outside the spindle, to achieve alignment (Figure 7).

Initiation of anaphase before full chromosome alignment but after significant mitotic delay indicates only a partial compromise of the spindle checkpoint in ZW10-depleted HEK293T or HeLa cells (Figures 2-3; Tables 1-2). Similar phenotypes are also observed in U2OS cells by Yang and colleagues, though the mitotic delay is milder [42]. Microinjection of anti-ZW10 antibody into HeLa cells also results in anaphase onset in the presence of misaligned chromosomes [32] Since loss of Mad2 from all kinetochores upon stable MT attachment is usually the hallmark of spindle checkpoint inactivation [2], the $\sim 20 \%$ kinetochore Mad2 left in ZW10-depleted cells (Figure 1) might be able to partially maintain the spindle checkpoint, thus resulting in the mitotic delay. Moreover, lack of dynein-mediated deprivation of kinetochore $\mathrm{Mad} 2$ and other spindle checkpoint proteins in these cells might further hinder inactivation of the checkpoint $[2,14]$.

\section{Acknowledgments}

The authors thank Qiongping Huang, Lirong Liu, and Wei Bian for technical assistance. We are grateful to Drs G Chan (Cross Cancer Institute, University of Alberta, Edmonton Alberta, Canada) for antibodies to human ZW10 and Rod, KH Choo (Murdoch Children's Research Institute, Royal Children's Hospital, Melbourne, Australia) for antiCREST serum, and E Fuchs (Rockefeller University, USA) for mRFP cDNA. This work was supported by the National Science Foundation of China (30330330, 30421005, and 30623003), Ministry of Science and Technology of China (2005CB522703 and 2007CB914501), and the Shanghai Municipal Council for Science and Technology (S048014317, 06DZ22032, and 058014578).

\section{References}

1 Rieder CL, Salmon ED. The vertebrate cell kinetochore and its roles during mitosis. Trends Cell Biol 1998; 8:310-318.

2 Cleveland DW, Mao Y, Sullivan KF. Centromeres and kinetochores: from epigenetics to mitotic checkpoint signaling. Cell 2003; 112:407-421.

3 Kapoor TM, Compton DA. Searching for the middle ground: mechanisms of chromosome alignment during mitosis. J Cell Biol 2002; 157:551-556.

4 Alberts B, D Bray, Lewis J, Raff M, Roberts K, Watson JD. Molecular Biology of the Cell. 3rd Edition. Garland Publishing Inc., 1994.

5 Hirokawa N. Kinesin and dynein superfamily proteins and the mechanism of organelle transport. Science 1998; 279:519-526.

6 Vallee RB, Williams JC, Varma D, Barnhart LE. Dynein: An ancient motor protein involved in multiple modes of transport. J Neurobiol 2004; 58:189-200.

7 King SJ, Schroer TA. Dynactin increases the processivity of the cytoplasmic dynein motor. Nat Cell Biol 2000; 2:20-24.

8 Karki S, Holzbaur EL. Cytoplasmic dynein and dynactin in cell division and intracellular transport. Curr Opin Cell Biol 1999; 11:45-53.

9 Gupta A, Tsai LH, Wynshaw-Boris A. Life is a journey: a genetic look at neocortical development. Nat Rev Genet 2002; 3:342355.

10 King JM, Hays TS, Nicklas RB. Dynein is a transient kinetochore component whose binding is regulated by microtubule attachment, not tension. J Cell Biol 2000; 151:739-748.

11 Echeverri CJ, Paschal BM, Vaughan KT, Vallee RB. Molecular characterization of the $50-\mathrm{kD}$ subunit of dynactin reveals function for the complex in chromosome alignment and spindle organiza- 
tion during mitosis. J Cell Biol 1996; 132:617-633.

12 Karess R. Rod-Zw10-Zwilch: a key player in the spindle checkpoint. Trends Cell Biol 2005; 15:386-392.

13 Starr DA, Williams BC, Hays TS, Goldberg ML. ZW10 helps recruit dynactin and dynein to the kinetochore. J Cell Biol 1998; 142:763-774.

14 Howell BJ, McEwen BF, Canman JC, et al. Cytoplasmic dynein/ dynactin drives kinetochore protein transport to the spindle poles and has a role in mitotic spindle checkpoint inactivation. J Cell Biol 2001; 155:1159-1172.

15 Yang ZY, Guo J, Li N, Qian M, Wang SN, Zhu XL. Mitosin/ CENP-F is a conserved kinetochore protein subjected to cytoplasmic dynein-mediated poleward transport. Cell Res 2003; 13:275-283.

16 Wojcik E, Basto R, Serr M, Scaerou F, Karess R, Hays T. Kinetochore dynein: its dynamics and role in the transport of the Rough deal checkpoint protein. Nat Cell Biol 2001; 3:1001-1007.

17 Liang Y, Yu W, Li Y, et al. Nudel Modulates Kinetochore Association and Function of Cytoplasmic Dynein in M Phase. Mol Biol Cell 2007; 18: 2656-2666

18 Maiato H, DeLuca J, Salmon ED, Earnshaw WC. The dynamic kinetochore-microtubule interface. J Cell Sci 2004; 117:54615477.

19 Merdes A, Heald R, Samejima K, Earnshaw WC, Cleveland DW. Formation of spindle poles by dynein/dynactin-dependent transport of NuMA. J Cell Biol 2000; 149:851-862.

20 Gaetz J, Kapoor TM. Dynein/dynactin regulate metaphase spindle length by targeting depolymerizing activities to spindle poles. J Cell Biol 2004; 166:465-471.

21 Maiato H, Rieder CL, Khodjakov A. Kinetochore-driven formation of kinetochore fibers contributes to spindle assembly during animal mitosis. J Cell Biol 2004; 167:831-840.

22 Vaisberg EA, Koonce MP, McIntosh JR. Cytoplasmic dynein plays a role in mammalian mitotic spindle formation. J Cell Biol 1993; 123:849-858.

23 Savoian MS, Goldberg ML, Rieder CL. The rate of poleward chromosome motion is attenuated in Drosophila zw10 and rod mutants. Nat Cell Biol 2000; 2:948-952.

24 Yan X, Li F, Liang Y, et al. Human Nudel and NudE as regulators of cytoplasmic dynein in poleward protein transport along the mitotic spindle. Mol Cell Biol 2003; 23:1239-1250.

25 Liang Y, Yu W, Li Y, et al. Nudel functions in membrane traffic mainly through association with Lis1 and cytoplasmic dynein. J Cell Biol 2004; 164:557-566.

26 van de Wetering M, Oving I, Muncan V, et al. Specific inhibition of gene expression using a stably integrated, inducible smallinterfering-RNA vector. EMBO Rep. 2003; 4:609-615.

27 Guo J, Yang Z, Song W, et al. Nudel Contributes to Microtubule Anchoring at the Mother Centriole and Is Involved in Both Dynein-dependent and -independent Centrosomal Protein Assembly. Mol Biol Cell 2006; 17:680-689.

28 Elbashir SM, Harborth J, Lendeckel W, Yalcin A, Weber K, Tuschl T. Duplexes of 21-nucleotide RNAs mediate RNA interference in cultured mammalian cells. Nature 2001; 411:494-498.

29 Kapoor TM, Mayer TU, Coughlin ML, Mitchison TJ. Probing spindle assembly mechanisms with monastrol, a small molecule inhibitor of the mitotic kinesin, Eg5. J Cell Biol 2000; 150:975988

30 Kops GJ, Kim Y, Weaver BA, et al. ZW10 links mitotic check- point signaling to the structural kinetochore. J Cell Biol 2005; 169:49-60.

31 Buffin E, Lefebvre C, Huang J, Gagou ME, Karess RE. Recruitment of Mad2 to the kinetochore requires the Rod/Zw10 complex. Curr Biol 2005; 15:856-861.

32 Chan GK, Jablonski SA, Starr DA, Goldberg ML, Yen TJ. Human $\mathrm{Zw}_{\mathrm{w}} 10$ and ROD are mitotic checkpoint proteins that bind to kinetochores. Nat Cell Biol 2000; 2:944-947.

33 Weaver BA, Bonday ZQ, Putkey FR, Kops GJ, Silk AD, Cleveland DW. Centromere-associated protein-E is essential for the mammalian mitotic checkpoint to prevent aneuploidy due to single chromosome loss. J Cell Biol. 2003; 162:551-563.

34 Yang Z, Guo J, Chen Q, Ding C, Du J, Zhu X. Silencing Mitosin Induces Misaligned Chromosomes, Premature Chromosome Decondensation before Anaphase Onset, and Mitotic Cell Death. Mol Cell Biol 2005; 25:4062-4074.

35 McEwen BF, Chan GK, Zubrowski B, Savoian MS, Sauer MT and Yen TJ. CENP-E is essential for reliable bioriented spindle attachment, but chromosome alignment can be achieved via redundant mechanisms in mammalian cells. Mol Biol Cell 2001; 12:2776-2789.

36 Yao X, Abrieu A, Zheng Y, Sullivan KF, Cleveland DW. CENP$\mathrm{E}$ forms a link between attachment of spindle microtubules to kinetochores and the mitotic checkpoint. Nat Cell Biol 2000; 2:484-491.

37 Kanda T, Sullivan KF, Wahl GM. Histone-GFP fusion protein enables sensitive analysis of chromosome dynamics in living mammalian cells. Curr Biol 1998; 8:377-385.

38 Williams BC, Gatti M, Goldberg ML. Bipolar spindle attachments affect redistributions of ZW10, a Drosophila centromere/kinetochore component required for accurate chromosome segregation. J Cell Biol 1996; 134:1127-1140.

39 Sasaki S, Shionoya A, Ishida M, et al. A LIS1/NUDEL/cytoplasmic dynein heavy chain complex in the developing and adult nervous system. Neuron 2000; 28:681-696.

40 Mayer TU, Kapoor TM, Haggarty SJ, King RW, Schreiber SL and Mitchison TJ. Small molecule inhibitor of mitotic spindle bipolarity identified in a phenotype-based screen. Science 1999; 286:971-974.

41 Sumara I, Gimenez-Abian JF, Gerlich D, et al. Roles of polo-like kinase 1 in the assembly of functional mitotic spindles. Curr Biol 2004; 14:1712-1722.

42 Yang Z, Tulu US, Wadsworth P, Rieder CL. Kinetochore Dynein Is Required for Chromosome Motion and Congression Independent of the Spindle Checkpoint. Curr Biol 2007; 17:973-980.

43 Lampson MA, Renduchitala K, Khodjakov A, Kapoor TM. Correcting improper chromosome-spindle attachments during cell division. Nat Cell Biol 2004; 6:232-237.

44 Mazumdar M, Misteli T. Chromokinesins: multitalented players in mitosis. Trends Cell Biol 2005; 15:349-355.

45 Rieder CL, Maiato H. Stuck in division or passing through: what happens when cells cannot satisfy the spindle assembly checkpoint. Dev Cell 2004; 7:637-651.

46 Putkey FR, Cramer T, Morphew MK, et al. Unstable Kinetochore-Microtubule Capture and Chromosomal Instability Following Deletion of CENP-E. Developmental Cell 2002; 3:351365 .

(Supplementary Information is linked to the online version of the paper on the Cell Research website) 